SAISIR L'ACTION DE LA COUR PENALE INTERNATIONALE PAR SA COMPLEMENTARITE

\title{
SEIZE THE ACTION OF THE INTERNATIONAL CRIMINAL COURT BY ITS COMPLEMENTARITY
}

Ovide E. Manzanga Kpanya

Université Fédérale de Kazan, Russie, Email: ovidemanzanga@gmail.com

Soumis: 11 Septembre 2019; Commentaire envoyé le: 14 Octobre 2019; Accepté: 31 Octobre 2019

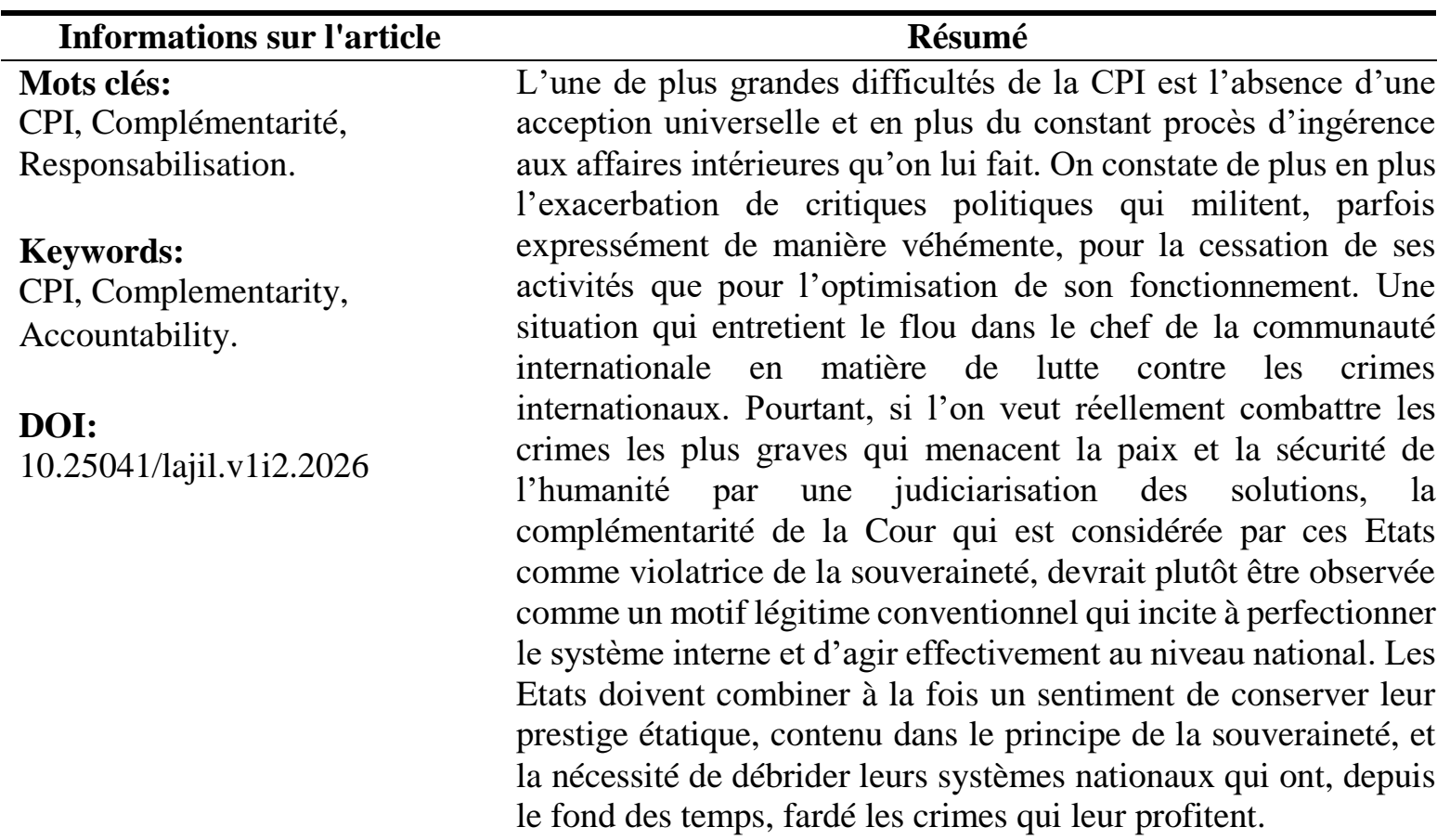

\begin{abstract}
Abstarct
One of the most significant difficulties of the ICC is the absence of a universal acceptance and, in addition to the constant process of interference in internal affairs in which it is made. We are increasingly seeing the worsening of political critics who advocate, sometimes expressly vehemently, to quit its activities rather than optimizing its functioning. This situation maintains the vagueness of the international community in the fight against transnational crimes. However, suppose we want to fight the most serious crimes that threaten the peace and security of humanity by judicializing solutions. In that case, the complementarity of the Court, which is considered by these States to violate sovereignty, should instead be observed as a legitimate conventional motive that incites to improve the internal system and act effectively at the national level. States must combine both a feeling of preserving their State prestige, contained in the principle of sovereignty and the need to unbridle their national
\end{abstract}


systems that have covered up the crimes that benefit them since the beginning of time.

\section{A. Introduction}

La conséquence juridique de l'existence de la CPI est le dédoublement juridictionnel dans le but de combattre à tous les niveaux certains de crimes les plus graves, dont les préjudices transcendent les frontières étatiques, dans lesquelles ils se commettent. Cependant, les deux niveaux d'instance ne peuvent agir concomitamment, évitant ainsi juridiquement, une situation permanente de litispendance, et politiquement, un conflit inévitable et persistant entre le statut de Rome et le principe de la souveraineté des Etats. Pour y remédier, le principe de la complémentarité a été jugé bien seyant, exprimant tout à fait le désir des États de contrôler leur système de droit pénal dans leurs propres pays, en particulier pour les crimes graves et ayant un contexte ou des conséquences politiques. ${ }^{1} \mathrm{Ce}$ souci débouchant principalement sur les considérations souverainistes, inextricables au positionnement juridico-politique des Etats, ne saurait néanmoins occulter l'avancée significative dans le mythe de la confiance qui doit jalonner l'idée de créer une Cour pénale internationale. Puisque dans cette reconfiguration de la souveraineté, ${ }^{2}$ ces Etats ont exprimé leur volonté encourageable ${ }^{3}$ de juridictionnaliser la réaction aux atrocités, dont la moindre justiciabilité doit, dorénavant, être exploitée.

En effet, il est important de signaler d'emblée, que ce caractère complémentaire de la Cour a d'abord été au centre de discussions de la commission chargée d'élaborer le statut, ${ }^{4}$ avant d'intéresser ensuite, vivement la doctrine, visiblement à cause de son caractère sui generis. Les analyses doctrinales qui ont consistées à dégager sa quintessence tendent toutes à la conclusion convergente, celle d'une Cour qui n'intervient qu'en cas d'absence de poursuites nationales, c'est-à-dire, ce n'est que lorsque l'Etat concerné n'a pas la capacité ou la volonté de le faire, que la Cour peut alors être compétente. ${ }^{5}$ Dans cette optique, la Cour pénale internationale s'observe comme un second niveau de souveraineté, qui redouble et englobe les souverainetés étatiques, lesquelles ne cessent pas pour autant d'exister, cela, dans un domaine régalien par excellence, celui de la justice pénale. ${ }^{6}$ Par voie de conséquence, en conservant son pouvoir, l'Etat demeure incontestablement, le seul premier sujet du droit international compétent pour engager les poursuites pénales à l'encontre de tous ceux qui transgressent sa loi pénale. Grosso modo, l'explication de la portée systémique ${ }^{8}$ de ce principe, c'està-dire ses aspects classique et positif, parait peu ou prou claire sur le rôle inéluctable de l'Etat en cette matière.

Pourtant dans la pratique, il existe des sérieux défis auxquels cette juridiction internationale doit courageusement affronter. D'une part, il s'agit de la méfiance à laquelle elle se heurte, venant de

\footnotetext{
${ }^{1}$ Paul SEILS, Guide de la complémentarité : Introduction quant au rôle des juridictions nationales et de la Cour pénale internationale dans la poursuite des crimes internationaux (ICTJ: Meredith Barges, 2016), 6.

${ }^{2}$ Philippe CURRAT, "HANS KELSEN, CARL SCHMITT ET LA COUR PENALE INTERNATIONALE. QUELQUES REFLEXIONS APRES LA PREMIERE DECENNIE D'EXERCICE DE LA COUR”, AFRI 14, (2013): 497-525, 498.

${ }^{3}$ La CPI est l'une de belles choses qui soient arrivées à la planète dans le domaine de réglementation des relations internationales. La résolution de juridictionnaliser les conflits généralement armés, détrône en réalité la guerre comme autrefois, moyen principal de résolution de ce conflits. "Comme indiqué dans la littérature scientifique, l'idée de créer la Cour pénale internationale incarnait les aspirations séculaires de l'humanité à un ordre mondial juste, impossible sans la punissabilité de crimes graves qui portent atteinte aux fondements de la civilisation humaine ". M.F. KHAMIDOVA, “CREATION D'UNE COUR PENALE INTERNATIONALE ET QUESTIONS JURIDIQUES DE RESPONSABILITE POUR LES CRIMES INTERNATIONAUX”, Bulletin de l'Université d'État de Saint-Pétersbourg 3, no. 8 (2017): 337 $348,337$.

${ }^{4}$ Alfiya. R KAYUMOVA, La juridiction pénale en droit international (Kazan: Centre d'innovation technologique, 2016) 313. V. Report of the ad hoc Committee on the Establishement of an International Criminal Court// Document de l'ONU du 06/09/1995.

${ }^{5}$ Voir les travaux de la Conférence de révision du Statut de Rome, tenue à Kampala, Ouganda, juin 2010, Complémentarité, $\mathrm{RC} / 11$, annexe $\mathrm{V}(\mathrm{c})$, Résumé officieux des points focaux, et autres documents liés, disponible sur le site Internet www.icccpi.int/iccdocs/asp_docs/RC2010/RC-11-Annex.V.c-FRA.pdf. Cf. également les Rapports du Bureau et du Secrétariat de l'Assemblée des Etats parties sur la complémentarité, présentés à la $11^{\text {ème }}$ session de l'Assemblée, La Haye, Pays-Bas, 1422 nov. 2011, respectivement ICC-ASP/11/24 et 25.

${ }^{6}$ Philippe CURRAT, Op.Cit., 2.

${ }^{8}$ Du principe de la complémentarité on constate deux aspects : le premier est classique basé sur la menace d'agir en lieu et place des juridictions nationales, et le second est positif en ce qu'il permet d'établir une certaine interaction entre la Cour pénale internationale et les systèmes nationaux.
} 
plusieurs membres de la communauté internationale ${ }^{9}$ parmi lesquels, on retrouve particulièrement les Etats-Unis, la Chine et la Russie, qui y sont par ailleurs membres du conseil de sécurité des nations unies, ${ }^{10}$ constituant ipso facto, une entrave redoutable dans l'acceptation universelle de cette juridiction. De fait, refusant initialement de reconnaitre cette juridiction, ces Etats ont, respectivement ou de manière solidaire, soulevés certaines objections notamment, contre la complémentarité de la Cour, qu'ils jugent violatrice de la souveraineté nationale. ${ }^{11}$ Certains d'ailleurs, les Etats-Unis en l'occurrence, n'hésitent pas de s'attaquer frontalement à la Cour, la qualifiant d'incapable et traitant ses juges de criminels, les menaçant de saisir leurs avoirs et de leur interdire l'accès aux USA. ${ }^{12}$ Des propos qui surviennent en guise de protestation contre la décision du Procureur de la Cour, d'ouvrir des enquêtes préliminaires sur les possibles crimes commis depuis le $1^{\mathrm{er}}$ mai 2003 en République islamique d'Afghanistan. ${ }^{13}$

D'autre part, elle s'achoppe à un sentiment de défiance, que l'on retrouve chez certains Etatsparties au statut de Rome, dont les ressortissants ${ }^{14}$ subissent ou sont menacé de subir l'action de la Cour. Mais aussi, les Etats eux-mêmes qui, paradoxalement, voient d'un mauvais œil les demandes de cette Cour concernant l'exécution des obligations contenues dans le chapitre XI du statut. A titre d'illustrations, la République des philippines qui pratiquait sa rude politique contre la drogue ${ }^{15}$, avait croisait sur son chemin le Procureur de la CPI, qui avait dénoncé une politique plutôt meurtrière soldée par des exécutions extra-judiciaires, susceptibles d'intéresser les enquêteurs de la Cour. ${ }^{16}$ N'ayant pas apprécié cette initiative, en 2018, le Président a révoqué la ratification de son pays du statut de Rome ${ }^{17}$. Mais il y a aussi, les Etats africains qui forment le groupe le plus important et, sont également en première ligne vers le portillon de sortie. En effet, la prouvabilité de leurs velléités individuelles n'est plus à démontrer, partant de la constatation des cas du Burundi, ${ }^{18}$ de la Gambie et

\footnotetext{
${ }^{9}$ La communauté internationale est pris ici au sens de H. Kelsen qui soutient qu'il n'y a de communauté qu'en tant qu'elle est constituée juridiquement. La communauté internationale est celle définie par l'ordre juridique international. Le Pacte de la Société des Nations est bien le fondateur de la communauté juridique internationale, comme le sera ensuite la Charte des Nations Unies. Philippe CURRAT, Op.Cit., 503.

${ }^{10}$ D'une part, en vertu de l'article 13 (b) du statut, le conseil de sécurité peut, agissant en vertu du chapitre VII de la Charte des Nations Unies, déférer au Procureur une situation dans laquelle un ou plusieurs de ces crimes paraissent avoir été commis. D'autre part, cet organe agit par des résolutions ayant théoriquement force obligatoires issues du vote, alors que les membres permanents du Conseil disposent chacun d'un droit de veto, pouvant bloquer toute initiative de déférer une
} situation au Procureur comme prévu.

${ }^{11}$ Steven W. BECKER, "LES OBJECTIONS DES GRANDES NATIONS A LA COUR PENAL INTERNATIONALE", ERES "Revue internationale de droit pénal" 81, no. 1 (2010): 47-64, 49.

${ }^{12}$ En 2018, La procureure de la CPI Fatou Bensouda avait annoncé aux médias en novembre qu'elle allait demander aux juges l'autorisation d'ouvrir une enquête sur des crimes de guerre présumés commis dans le cadre du conflit afghan, notamment par l'armée américaine. John Bolton, Conseiller à la sécurité nationale de la Maison-Blanche, n'a pas loupé l'occasion de lui répondre au nom du Président Trump en ces termes: "Nous allons interdire à ces juges et procureurs l'entrée aux Etats-Unis. Nous allons prendre des sanctions contre leurs avoirs dans le système financier américain et nous allons engager des poursuites contre eux dans notre système judiciaire". "Nous n'allons pas coopérer avec la CPI, nous n'allons pas lui fournir d'assistance, nous n'allons pas adhérer à la CPI. Nous allons laisser la CPI mourir de sa belle mort» car «pour nous, la CPI est déjà morte". Voir l'article dans le magazine LE TEMPS, Publié lundi 10 septembre 2018 à 19:51, modifié lundi 10 septembre 2018 à 19:54.

${ }^{13}$ Déclaration du 20 Novembre 2017, Le Procureur de la Cour pénale internationale, Fatou Bensouda, demande aux juges l'autorisation d'ouvrir une enquête concernant la situation en République islamique d'Afghanistan. https://www.icccpi.int/about? $\ln =\mathrm{fr}$

${ }^{14}$ Conformément à l'article premier du statut de Rome, la cour comme juridiction internationale, ne juge pas les Etats mais plutôt leurs ressortissants. Lire aussi ORENTLICHER, Diane F, "POLITIQUE PAR D'AUTRES MOYENS: LE DROIT DE LA COUR PENALE INTERNATIONALE”, Cornell International Law Journal 32, no. 3 (1999).

${ }^{15}$ L'équipe de pays des Nations Unies, se référant à des recommandations ayant reçu l'aval des Philippines, a indiqué que la campagne contre la drogue menée par le Gouvernement s'était soldée par l'exécution de prétendus "toxicomanes et revendeurs de drogue". V. Rapport du Haut-Commissariat des Nations Unies aux droits de l'homme du Conseil des droits de l'homme Groupe de travail sur l'Examen périodique universel, Vingt-septième session 1 er -12 mai 2017, Compilation concernant les Philippines, 3.

${ }^{16}$ Déclaration du Procureur de la Cour pénale internationale, Mme Fatou Bensouda, à propos de l'ouverture d'un examen préliminaire dans le cadre des situations aux Philippines et au Venezuela, du 8 February 2018, Bureau du Procureur de la CPI : https://www.icc-cpi.int/Pages/item.aspx?name=180208-otp-stat\&ln=fr

${ }^{17}$ La République des Philippines a déposé, le 17 mars 2018, la notification écrite de son retrait du Statut de Rome, le traité fondateur de la Cour, auprès du Secrétaire général des Nations Unies en tant que dépositaire du Statut. RéférenceC.N.138.2018.TREATIES-XVIII.10//https://treaties.un.org/doc/publication/cn/2018/cn.138.2018-eng.pdf

${ }^{18}$ Le 12 octobre 2016, le Parlement de la République du Burundi a adopté une loi en faveur du retrait de son pays du Statut de Rome (Statut), traité fondateur de la Cour pénale internationale (CPI). Depositary Notification 
de de l'Afrique du sud ${ }^{19}$, et tant d'autres Etats qui ont menacé de quitter la Cour. Mais le cas qui parait quelque peu atypique, est celui d'une action collective portée par l'Union africaine, qui, dans l'affaire soudanaise, avait demandé aux Etats membres africains de ne pas coopérer avec la Cour. ${ }^{20}$ En outre, dans l'optique de s'en débarrasser, cette principale organisation régionale avait, en 2014, adoptée le protocole de Malabo, se rapportant à la création d'une chambre criminelle à la Cour africaine des droits de l'homme et des peuples ${ }^{21}$ chargée de connaitre les affaires pénales africaines.

Dans tous les cas, toutes ces attaques, critiques, objections ou observations dont les fonds pourraient faire l'objet d'une analyse à part entière, démontrent formellement, la difficulté de l'universalisation de cette Cour et surtout, l'incompréhensibilité de son caractère subsidiaire, tout en fulminant son caractère permanent donc, sa stabilité, y compris, sa crédibilité. Somme toute, la question subséquente qui traverse l'esprit, est celle de savoir, à quoi sert réellement cette juridiction que l'on désire et décrie à la fois? Sans nul doute, une relecture téléologique du principe de la complémentarité serait l'hypothèse la plus plausible afin de mieux rencontrer et décortiquer cette forme de binarité qu'il renferme. Pour ce faire, le premier point sera consacré à la responsabilisation de l'Etat dans la répression de ces crimes internationaux, laquelle renvoie d'une part, à la priorité qu'ont les juridictions nationales, et d'autre part, à la capabilisation du système judiciaire étatique, au cas où, ceci serait jugé défaillant. Le second point se présentera, tel un corollaire du premier, en ce qu'il abordera l'intervention de la Cour dans un Etat donné, lorsque les conditions statutaires auront suffisamment été constatées.

\section{B. Discussion}

\section{La responsabilisation de l'Etat dans la répression des crimes internationaux}

Si l'éclosion de cette instance a été provoquée à la suite du constat de la défaillance ou du laxisme permanent des juridictions étatiques, sa complémentarité impliquerait donc une capabilité de ces instances nationales (B), comme condition sine qua non de leur priorité d'agir (A).

\section{a. Priorisation des juridictions nationales}

L'article premier en combinaison avec l'avant dernier paragraphe du préambule du statut de Rome déterminent pleinement la nature de cette Cour, qui, à la différence d'autres juridictions pénales internationales $a d h o c$, fonde son existence sur sa complémentarité des juridictions pénales nationales. ${ }^{22}$ Cette complémentarité que l'on qualifie de fondamentale, a été l'une des conditions préalables ${ }^{23}$ de son acceptation par les Etats-signataires, s'apercevant alors comme un équilibre ou un compromis entre le respect de la souveraineté et le développement d'une institution judiciaire autonome et indépendante ${ }^{24}$ sur le plan international.

\footnotetext{
C.N.805.2016.TREATIES-XVIII.10 // United Nations Treaty Collection. URL: https://treaties.un.org/doc/Publication/CN/2016/CN.805.2016-Eng.pdf

${ }^{19}$ En novembre 2016, la Gambie sous l'ère JAMEH, avait notifié au Secrétaire général de l'ONU son retrait de la CPI, alors qu'à l'aube de l'élection d'Adama BARROW, le nouveau président a décidé d'annuler la décision de retrait du pays du statut de la Cour pénale internationale par la Gambie, par une lettre officielle envoyée au Secrétaire général des Nations Unies le 10 janvier 2017 le Ministre des affaires étrangères, de la coopération internationale et des Gambiens à l'étranger. La décision du président sud-africain ZUMA a depuis été déclarée "inconstitutionnelle" par la Haute Cour à Pretoria Depositary Notification C.N.862.2016.TREATIES-XVIII.10 // United Nations Treaty Collection. URL: https://treaties.un.org/doc/Publication/CN/2016/CN.862.2016-Eng.pdf

${ }^{20}$ Conférence de l'Union Africaine, 13e session ordinaire, Syrte, 3 juillet 2009, Décision sur le rapport de la réunion des États africains parties au Statut de Rome de la Cour pénale internationale (C.P.I.) - Assemblée/AU/Déc., 245 (XIII) Rev.1, $\S 10$.

${ }^{21}$ En juin 2014, le Protocole portant amendements au Protocole portant Statut de la Cour africaine de justice et des droits de l'homme (ci-après le protocole de Malabo) a été adopté par l'Union africaine. Le Protocole de Malabo prévoit d'intégrer dans le mandat de la Cour africaine de justice et des droits de l'homme (CAJDH) une compétence en matière pénale.

${ }^{22}$ Otto TRIFFTERER, Commentaire du Statut de Rome de la Cour pénale internationale, Observations, notes article par article, 1 ère éd., (Bruxelles: Bruylant, 1999) 59. Cité par Almoktar ASHNAN, "Le principe de complémentarité entre la cour pénale internationale et la juridiction pénale nationale", Thèse, Faculté de Droit/Droit public, Université François Rabelais de Tours, (2015), 19

${ }^{23}$ Svetlana Y. KORABLEVA, Régime de complémentation de la cour pénale internationale: problèmes de mise en œuvre, document version russe en ligne sur : http://www.elibrary.az/docs/jurnal/jrn2017_731.pdf, consulté le 20/01/2019.

${ }^{24}$ Almoktar ASHNAN, Op.Cit., 22.
} 
Faut-il rappeler, qu'insistant sur le fait que la répression de toute criminalité, quelle que soit l'échelle, relève de la mission régalienne de tout Etat, qui la remplit conformément à sa législation, le principe de la complémentarité incite les Etats à agir eux-mêmes et, adéquatement. Il rappelle donc en d'autres mots, cette nécessité selon laquelle, il est du devoir de chaque État de soumettre à sa juridiction criminelle les responsables de crimes internationaux. ${ }^{25} \mathrm{C}$ 'est à la fois un engagement politique et juridique d'un Etat, d'un gouvernement de faire régner la justice sur son territoire, et pour y parvenir, les mesures effectives doivent être prises dans le cadre national en vue de réprimer ces crimes, de poursuivre leurs auteurs et d'en prévenir efficacement. On peut mentionner par exemple, le renforcement des cadres normatif et structurel; la disponibilisation du personnel judiciaire capable d'assurer cette tâche délicate; l'élaboration d'une politique pénale adéquate qui martèle sur le caractère prioritaire de la répression de ces crimes, sans ignorer la mise en place d'un système pénitentiaire assurant, en cas de condamnation, l'effectivité de l'exécution de la peine et la perceptibilité de ses effets notamment, dissuasifs.

Pourtant, cet aspect de la complémentarité de la Cour est largement ignoré par une grande majorité de l'opinion publique, qui voit en l'action de cette Cour une main noire impérialiste, néocolonialiste, une juridiction contre les Etats faibles. Certains évoquent un tropisme africain, ${ }^{26} \mathrm{en}$ référence aux nombreuses affaires concernant les africains qui abondent son agenda. Concrètement, il s'agit plus d'une critique purement politique que l'on adresse à la Cour qui n'est d'ailleurs pas surprenante, lorsqu'on prend en considération le constant rapport de force teinté du souverainisme qui caractérise le droit international, sur lequel, l'influence est indéniable. Cependant, abonder dans le même sens, ce serait apprécier incorrectement la notion de la complémentarité du point de vue juridique. Puisqu'en effet, ce principe exhorte les Etats à affirmer leurs capacité et la volonté de mener les poursuites à l'encontre des personnes qui se rendraient coupables de l'un ou plusieurs de ces crimes prévus à l'article 5 du statut de Rome. A défaut de ces prétentions, la Cour intervient car, sa complémentarité la rendrait, dans cette hypothèse, garante des procédures et poursuites nationales.

Il ne s'agit pas, comme le pensent certains auteurs, d'une relation verticale entre les États et la $\mathrm{CPI}$, plaçant cette dernière dans un rôle d'instance judiciaire de dernier recours décidant de la recevabilité ou non d'une affaire..$^{29}$ Car la complémentarité qui nourrit cette juridiction ne saurait renfermer un autre sens que celui de responsabiliser les Etats, les encourager à déployer des efforts possibles qui permettent de mettre en place un système répressif à la hauteur de ces infractions. A ce compte-là, il ne peut exister une verticalité dans la relation qui relie la Cour aux Etats. On y aperçoit par contre, une horizontalité passive, un parallélisme non-concurrentiel qui place néanmoins, les juridictions nationales en priorité d'action, et qui les privilégie en cas de conflit. ${ }^{30}$

Par ailleurs, si du régime de la complémentarité ressortent deux juridictions compétentes, il y a des raisons de croire, que l'existence simultanée d'une instance pénale (internationale) permanente à côté de celle nationale soit de nature à menacer le prestige étatique, puisqu'elle est en quelque sorte, révélatrice et symptomatique des insuffisances et incapacités d'un Etat, d'un gouvernement, d'un régime politique à mobiliser les ressources nécessaires pour mettre en place une justice qui assure et rassure la stabilité de sa société. En plus, comme il a été souligné précédemment, la nécessité de cette Cour a été fortement ressentie par le constat général d'après lequel, les justices nationales ont toujours fait preuve d'un certain laxisme notoire en matière de crimes internationaux eu égard à leur caractère plus ou moins politique. Conséquemment, les Etats ont toujours eu tendance, jusqu'à ce jour, à grimer ceux des crimes qui leur profitent.

Toutefois, il sied de relever aussi, que cette juridiction souffre de la faiblesse du droit international qui est en réalité, une branche juridique largement émaillée de tendances politiques diverses fortement mouvantes. Les constantes négociations politiques qui fondent en réalité cette branche du point de vue de sa source principale, présentent un grand inconvénient, qui est la

\footnotetext{
${ }^{25} 10^{\text {ème }}$ paragraphe du préambule du statut de Rome.

26 Sara DEZALAY, "L'AFRIQUE CONTRE LA COUR PENALE INTERNATIONALE ? ÉLEMENTS DE SOCIOGENESE SUR LES POSSIBLES DE LA JUSTICE INTERNATIONALE”, Editions Karthala Politique africaine 156, no. 2 (2017): 165-182, 168.

${ }^{29}$ Maxime C.-TOUSIGNANT, "L'INSTRUMENTALISATION DU PRINCIPE DE COMPLEMENTARITE DE LA CPI: UNE QUESTION D'ACTUALITE”, Revue Québécoise de droit international 25, no. 2 (2012): 73-99, 88. https://www.icc-cpi.int/NR/rdonlyres/1FA7C4C6-DE5F-42B7-8B25 60AA962ED8B6/143595/030908_Policy_Paper_FR.pdf., Consulté le 30/01/2019.
} 
succombance du plus faible, de la nation la moins puissante, la moins soutenue, ${ }^{31}$ car il s'agit d'un rapport de force évidemment défavorable à l'un ou l'autre. Ce caractère très poisseux au droit international, épargne très faiblement la Cour, qui, lors de l'appréciation de la recevabilité d'une affaire, peut s'exposer à une sorte d'instrumentalisation politique tant sur le plan national ${ }^{32}$ qu'international. ${ }^{33}$ Néanmoins, comme le souligne bien Kelsen, le droit international, comme tout système, ne suppose pas une subordination politique c'est-à-dire, des êtres humains qui appliquent la loi par rapport à ceux qui l'édictent. Il est avant tout un dispositif de subordination juridique des êtres humains aux règles légales : non sub homine sed sub lege. ${ }^{34} \mathrm{~A}$ cet effet, s'appuyant sur cette responsabilisation que renferme la complémentarité (comme une norme juridique), l'Etat se doit de capabiliser son système judiciaire national en vue de s'abriter juridiquement contre une quelconque intervention qu'il pourrait juger malveillante ou violatrice de sa souveraineté, car dans tous cas, ces crimes devraient être réprimés.

\section{b. Implication fondamentale de la responsabilisation: Capabilisation du système judiciaire national face aux crimes relevant de la compétence de la Cour}

On compte nombreux Etats politiquement stables, qui ont des systèmes judiciaires consolidés par leurs stabilités et leur sens profond d'Etat de droit. Mais il y a aussi d'autres, précisément dans des régions à fortes fluctuations socio-politico-militaires, qui accusent beaucoup de difficultés susceptibles de tétaniser toute action répressive sérieuse. Dans ce cas, la responsabilisation de l'Etat soumet principalement ces Etats à une capabilisation de leurs systèmes judiciaires, parce qu'il serait utopique de prétendre réprimer les crimes d'une telle envergure lorsque la justice, comme organe légitime chargé de la répression, est visiblement défaillante ou inexistante. C'est-à-dire que, ces Etats ne doivent pas demeurer dans le statu quo, en se confiant en l'action de la CPI. Le faire, ce serait transgresser sans nul doute l'esprit et la lettre du statut de Rome, spécialement, en son article premier. D'ailleurs, le fait qu'un grand nombre d'affaires atteignent la Cour ne devrait pas constituer un critère d'efficience de la complémentarité 35 ni de la Cour. L'efficacité de la CPI, c'est l'absence de procès, telle une conséquence du fonctionnement efficace des systèmes judiciaires nationaux. ${ }^{36}$

En réalité, certains Etats agissent dans ce sens, notamment, la Libye, qui, après le déboulonnement du régime du Colonel Mouammar Kadhafi, avait consentie beaucoup d'efforts pour restaurer l'Etat de droit, et enfin prétendre avoir la capacité de juger ses propres ressortissants. Ce qui lui a valu d'ailleurs une ''victoire judiciaire" sur le Procureur de la CPI dans l'affaire Abdullah Al-Senoussi ${ }^{37}$. Il y a aussi le cas de la République Démocratique du Congo, le soixantième

\footnotetext{
${ }^{31}$ On peut illustrer par les cas soudanais et syrien devant le conseil de sécurité. Deux situations qui auraient pu subir le même sort, ne l'ont finalement pas été parce que, l'une, protégée par un organe régional dépourvu de toute force "contraignante" et l'autre par un Etat (la Russie) et ses alliés (notamment la Chine) possédant un moyen dirimant de toute décision du conseil de sécurité (droit de véto).

${ }^{32}$ Au niveau interne, les Etats qui camouflaient les crimes qui leur profitaient n'ont pas renoncés à leur attitude mais, l'ont enveloppée par la complémentarité. En effet, pour se débarrasser des opposants politiques gênants, certains dirigeants politiques au pouvoir n'hésitent pas à recourir à la cour pénale internationale en vertu de la complémentarité, tel est le cas de la République Démocratique du Congo, qui plaida la défaillance et l'incapacité du système congolais à l'époque pour saisir la Cour avec un espoir non-apparent de mettre en difficulté quelques personnalités politiques qui représentaient un risque pour le pouvoir en place. On peut également citer, le cas de la cote d'ivoire dont seuls Gbagbo et Blé Goudé ont été transféré à Scheveningen, alors que les mêmes autorités ont refusé le transfèrement de Simon Gbagbo, qui a bénéficiée en outre d'une grâce présidentielle après des poursuites internes.

${ }^{33}$ Sur le plan international, le conseil de sécurité saisit la Cour en vertu de l'article...ne manque pas l'occasion d'afficher ses divergences à chaque fois qu'il faut user de ces pouvoirs que lui confère le statut de Rome. L'affaire syrienne en est l'exemple concret couvrant les violations graves du DIH et des droits de l'homme.

${ }^{34}$ GRANGÉ, Ninon (dir.), RAMEL, Frédéric (dir.), Le droit international selon Hans Kelsen : Criminalités, responsabilités, normativités. Nouvelle édition [en ligne], (Lyon: ENS Éditions, 2018) (généré le 24 mars 2019). Disponible sur Internet : <http://books.openedition.org/enseditions/8716>. ISBN : 9791036200090. DOI : 10.4000/books.enseditions.8716.

${ }^{35}$ Almoktar ASHNAN, Op.Cit., 20.

${ }^{36}$ Communication relative à certaines questions de politique générale concernant le Bureau du Procureur. Document en ligne sur: https://www.icc-cpi.int/NR/rdonlyres/1FA7C4C6-DE5F-42B7-8B25 60AA962ED8B6/143595/030908_Policy_Paper_FR.pdf. (Consulté le 30/01/2019)

${ }^{37}$ Le Procureur c. Saif Al-Islam Kadhafi et Abdullah Al-Senussi : Le 11 octobre 2013, la Chambre préliminaire I de la Cour pénale internationale (CPI) a décidé que l'affaire concernant Abdullah Al-Senussi faisait l'objet d'une enquête nationale par les autorités libyennes compétentes et que ce pays avait la volonté et était capable de mener véritablement à bien cette enquête. Par conséquent, les juges ont conclu que l'affaire était irrecevable devant la Cour eu égard au principe de
} 
Etat à ratifier le Statut de Rome ${ }^{37}$, ayant permis par là son entrée en vigueur, le premier Etat à voir l'un de ses ressortissants traduit devant la Cour ${ }^{38}$, et à ce jour, elle compte le plus grand nombre de ressortissants qui ont été ou sont jugés par devant cette Cour. Une observation qui ne peut que susciter une certaine curiosité sur la capacité du système judiciaire de cet Etat en matière de crimes internationaux.

En effet, ces infractions ne sont pas inconnues de la législation congolaise. Le texte y relatif remonte bien à l'Ordonnance-loi n ${ }^{\circ} 72 / 060$ du 25 septembre 1972 portant institution d'un Code de justice militaire, qui prévoyait en son chapitre VI du titre II du livre III les crimes de guerre et contre $1^{\prime}$ 'humanité ${ }^{39}$. En 2002, quelques mois après l'entrée en vigueur du statut de Rome, et dans un contexte de conflits armés accablant le pays, deux nouvelles lois ont vues le jour, se rapportant respectivement, au code pénal militaire ${ }^{40}$ et au code judiciaire militaire ${ }^{41}$. Ces textes de nature essentiellement militaire, posaient un apparent problème quant à l'aptitude de juridictions militaires à connaitre de ces crimes, au cas où leurs auteurs eurent été des civils ${ }^{42}$. Car, on y voyait visiblement une certaine exclusivité compétencielle conférée aux juridictions militaires ${ }^{43}$, qui devraient appliquer lesdits textes. Une situation qui a en outre, révélée la porosité du système judiciaire congolais face aux enjeux de ces crimes particuliers, et par conséquent la nécessité de relever le défi de l'efficacité et de la pleine compétence dudit système. A cet effet, deux réformes méritent d'être soulevées :

La première est celle de $\mathbf{2 0 1 3}^{\mathbf{4 4}}$ relative à l'organisation judiciaire, qui a le mérite d'attribuer pour la première fois, à une juridiction de droit commun, précisément la Cour d'appel, une compétence personnelle élargie afin de connaitre de crimes prévus à l'article 5 du statut de Rome. En effet, l'article 91 en son alinéa 2 point 1 de la loi relative à l'organisation et fonctionnement des juridictions de l'ordre judiciaire, dispose que: les 'Cours d'appel connaissent aussi du crime de génocide, des crimes de guerre et des crimes contre l'humanité commis par les personnes relevant de leur compétence et de celle des ribunaux de grande instance". La privation du TGI de ses justiciables personnels qui conduit à la turgescence de la compétence des Cours d'appel entant que juge du premier degré, est en quelque sorte une innovation apportée par cette disposition, qui n'a pourtant pas échappée à diverses interprétations, dont celle qui, basée sur sa combinaison avec l'article 27 du statut, tend à leur reconnaitre l'exclusivité de la compétence en la matière.

Rencontrant avec clarté les difficultés d'interprétation soulevées par cette disposition, le Professeur Wane, dans un article publié en 2018, estime que cette disposition consacre à la fois une affirmation de la compétence des cours d'appel et, une non-infirmation de la compétence des autres juridictions en matière de crimes contre la paix et la sécurité de l'humanité. Cette position est justifiée par le fait que, l'art 27 précité, qui réfute toute procédures spéciales notamment, le privilège de juridiction, est applicable uniquement à la CPI et non en droit interne, et ce, conformément aux libellés dudit article en combinaison avec l'article premier du statut. ${ }^{45}$

complémentarité consacré par le Statut de Rome, traité fondateur de la CPI. https://www.icccpi.int/Pages/item.aspx?name=pr1034\&ln=fr

${ }^{37}$ La République démocratique du Congo a ratifié cette Convention internationale à travers son Décret $\mathrm{n}^{\circ} 0013$ du 30/03/2002.

${ }^{38}$ Affaire : Le Procureur $c$. Jean-Pierre Bemba Gombo

39 Articles 522, 523 et 530 de cette ordonnance-loi prévoyant respectivement l'empoisonnement des eaux et denrées consommables, la mise à mort par représailles et le génocide. V. Bienvenu WANE BAMEME, "la question de juridictions congolaises compétentes en matière de crimes contre la paix et la sécurité de l'humanité", Revue de l'université congolaise I, 57 (2017): 55-83.

${ }^{40}$ La loi $\mathrm{n}^{\circ} 024 / 2002$ du 18 novembre 2002 portant Code pénal militaire

${ }^{41}$ Loi n ${ }^{\circ} 023 / 2002$ du 18 novembre 2002 portant Code judiciaire militaire

${ }^{42}$ Ces crimes (internationaux) étaient classés en effet plutôt parmi les infractions mixtes (infractions de droit commun mais aggravées), on peut en comprendre qu'ils pouvaient être commis par quiconque (comme c'est d'ailleurs le cas concrètement) et de ce fait, être portés devant toute juridiction compétente (ordinaire ou spécialisée). Lire le Professeur Bienvenu WANE BAMEME, Op.Cit., 40.

${ }^{43}$ La loi de corroborée par de la loi $n^{\circ} 15 / 023$ du 31 décembre 2015 modifiant la loi $n^{\circ}$ 024-2002 du 18 novembre 2002 portant Code pénal qui, dans le deuxième paragraphe de son préambule soutient que, la reconnaissance par la loi organique de 2013, aux juridictions de droit commun, la compétence de connaitre de ces crimes, leur eut perdu le caractère d'infraction exclusivement militaire.

${ }^{44}$ Loi organique ${ }^{\circ} 13 / 011-\mathrm{B}$ du 11 avril 2013 portant organisation, fonctionnement et compétences des juridictions de l'ordre judiciaire, in JO RDC,54ième année, $\mathrm{n}^{\circ}$ spécial-Première partie, du 04 mai 2013.

${ }^{45} \mathrm{La}$ Cour dont il est question à l'article 27 point 2, il ne peut s'agir d'une autre Cour que celle prévue par l'article premier du statut de Rome. 
C'est ainsi qu'en République Démocratique du Congo, à travers l'éclairage apporté par la doctrine dans l'interprétation de cette réforme, la répartition de compétences en matière de ces crimes est très étendue, impliquant une panoplie de juridictions notamment, le Tribunal de grande instance qui conserve sa compétence d'attribution ${ }^{46}$; la Cour d'appel qui juge ses justiciables et ceux du Tribunal de grande instance ${ }^{47}$; la Cour de cassation qui poursuit ses justiciables ${ }^{48}$; la Cour constitutionnelle siégeant en matière pénale pour connaitre de l'infraction commise par le président de la République et le premier ministre ${ }^{49}$; le Tribunal pour enfant, seule juridiction compétente en matière d'infractions commises par les personnes âgées de moins de 18 ans,${ }^{50}$ et sans ignorer les juridictions militaires. ${ }^{51}$

La seconde réforme est celle de 2015 traitant de la coopération judiciaire avec la CPI":52 La relation qu'entretienne cette Cour avec chaque Etat est avant tout diplomatique, et de celle-ci, s'établit la coopération judiciaire. En ce sens, pour exercer sa mission, elle s'appuie pleinement sur la justice étatique pour toute procédure judiciaire dont elle estime nécessaire. En réalité, l'ouverture d'une enquête par la CPI nécessite donc, une investigation qui impliquerait forcément, une entraide judiciaire, entre la justice de l'Etat dans lequel se trouvent les renseignements jugés indispensables pour l'enquête; 'une arrestation et une remise du suspect" dans le cas où la chambre préliminaire aura fait droit à la requête du procureur conformément à l'article 58 point 1 du statut; une exécution de la peine qui, en principe, s'effectue dans l'un des Etats parties qui l'accepte.

Il va sans dire que, la coopération entre la Cour et les Etats est un aspect primordial du fonctionnement de cette juridiction parce que, sans elle, il lui est impossible de mettre en branle ses actions. Il existe pour ce faire, une obligation de coopérer (c'est-à-dire un consentement donné lors de la ratification ou de la reconnaissance de la compétence de la Cour en vertu du principe pacta sunt servanda) et une forme d'exhortation pour les autres Etats, car, n'étant pas liés, ils ne sont pas tenus de coopérer. C'est ainsi que pour assurer la pleine collaboration avec la Cour quelle que soit la forme de l'implémentation du statut en droit interne, chaque Etat partie veille à prévoir dans sa législation nationale les procédures qui permettent la réalisation de toutes les formes de coopération visées par le statut $t^{53}$. On peut lire entre les lignes de cette obligation, une importante forme de la responsabilisation de l'Etat, consistant à le confronter avec sa propre législation nationale (tu patere legem quam ipse fecisti) (même si ceci parait moins suffisant pour contrecarrer pratiquement une éventuelle position politique dissidente).

Cette recommandation a été prise en compte par le législateur congolais à travers cette réforme, qui précise clairement l'organe habilité de coopérer avec la Cour pénale internationale à savoir, le Procureur général près la Cour de cassation intervenant dans tous les cas généraux, et le Procureur général près la Cour constitutionnelle qui agit que lorsqu'il s'agit des justiciables de cette Cour. ${ }^{54}$ Ainsi, lorsque le Procureur de la Cour pénale internationale souhaite intervenir directement sur le territoire de cet Etat, il en avise immédiatement le Procureur général concerné. Ce dernier peut, en réponse à une demande d'entraide judiciaire, faire valoir des préoccupations et proposer au Procureur de la Cour pénale internationale d'exécuter lui-même ces actes, s'ils peuvent être exécutés dans les mêmes délais et selon les mêmes modalités. ${ }^{55}$

En pratique, dans le cadre de l'entraide judiciaire, la justice congolaise peut, sur demande de la Cour, procéder au rassemblement d'éléments de preuve, y compris aux dépositions faites sous serment, à la production d'éléments de preuve, aux expertises et rapports dont la Cour a besoin, et ce, conformément aux pouvoirs du procureur de la CPI d'enquêter sur le territoire d'un Etat ${ }^{56}$. Dans

\footnotetext{
${ }^{46}$ Article 89 al. $1^{\text {er }}$ de la loi organique ${ }^{\circ} 13 / 011-B$ précitée.

${ }^{47}$ Article 91 de la loi organique $\mathrm{n}^{\circ} 13 / 011-\mathrm{B}$ précitée.

${ }^{48}$ Article 93 de la loi organique $\mathrm{n}^{\circ} 13 / 011$-B précitée.

${ }^{49}$ Art. 163 et 164 de la Constitution du 18 février 2006 telle que modifiée par la loi $n^{\circ} 11 / 002$ du 20 janvier 2011 portant révision de certains articles de la constitution, in J.O.RDC., $52^{\text {ème }}$ année, $\mathrm{n}^{\circ}$ spécial du 05 février 2011.

${ }_{50}$ Art. 94 et 99 al. 1 de la loi n ${ }^{\circ} 09 / 001$ du 10 janvier 2009 portant protection de l'enfant, in JO RDC, 50è année, $n^{\circ}$ spécial, Première partie, du 12 janvier 2009

${ }^{51}$ Lire à ce sujet le Professeur B. WANE BAMEME, Op.Cit., 40.

${ }^{52}$ Loi n $^{\circ} 15 / 024$ du 31 décembre 2015 modifiant et complétant le Décret du 06 août 1959 portant Code de procédure pénale.

${ }^{53}$ Article 88 du statut de Rome.

${ }^{54}$ Article 21 bis $\$ 3$ de loi $n^{\circ} 15 / 024$ du 31 décembre 2015 précitée.

${ }^{55}$ Article 21-11e de loi $\mathrm{n}^{\circ} 15 / 024$ du 31 décembre 2015 précitée.

${ }^{56}$ Article 54 point 3 du statut de Rome précité.
} 
le même ordre d'idée, en matière d'interrogatoire, conformément aux articles 54, 86, 93 du statut, le procureur a le pouvoir d'interroger toute personne dont il estime l'audition nécessaire, mais il peut solliciter à cette fin, le concours des autorités judiciaire d'un Etat. Ainsi, en vertu de l'article 86 du statut et de l'article 21-13 $3^{\text {ème }}$ du code de procédure pénale congolaise, la justice congolaise peut, sur demande de la Cour, procéder à cet interrogatoire. Il en est de même en matière d'arrestation dont les demandes émanant de la Cour sont adressées au même organe, en original et en copie certifiée accompagnées de toutes les pièces justificatives ${ }^{57}$. Ces mandats d'arrêt sont exécutés ${ }^{58}$ par le procureur général concerné et ce, conformément à la légalité nationale en vigueur.

Enfin, il faut bien en prendre garde, car les deux aspects de réformes décrits ci-haut, ne sauraient suffire à eux-seuls, car il faut qu'il y ait en outre, une volonté étatique manifeste et agissante afin de rendre effective la répression, a contrario, une action supplétive s'avérerait nécessaire.

\section{L'intervention supplétive de la Cour pénale internationale}

L'intervention de la Cour tire son fondement des articles 13 et 15, lesquels sont soumis aux conditions de recevabilité prévues à l'article 17 (A), tendant clairement à faire application du principe de la complémentarité. Toutefois, le fait d'établir les conditions de recevabilité ne sert pas de saufconduit à la Cour d'agir sur le territoire de l'Etat indiqué, puisque l'obligation de notification prévue à l'article 18 (B) révèle une autre forme de subsidiarité, qui privilégie encore une fois l'action de l'Etat dans la mesure où, elle lui permettrait de se saisir d'une affaire qui semblait éluder sa diligence.

\section{a. Conditions de recevabilité prévues par l'art 17 du statut de Rome: Constat d'une prévisibilité de l'impunité}

L'analyse de l'article 17 du statut permet de considérer l'intervention conditionnelle de la Cour comme le second aspect de la complémentarité, basé sur le manque de volonté (1) et ou l'incapacité (2) d'un Etat à réprimer ces crimes internationaux.

1) Appréciation du manque de volonté

En considération du procès équitable tel que reconnu en droit international, l'appréciation du manque de volonté se fonde sur trois cas de figures:

Premièrement, il doit s'agir d'une procédure engagée ou déjà close ou de la décision de l'État prise dans le dessein de soustraire la personne concernée de sa responsabilité pénale pour les crimes relevant de la compétence de la Cour. En d'autres termes, il doit s'agir d'une procédure ou d'une décision de l'Etat ayant pour but de protéger le suspect contre une action judiciaire internationale. La chambre préliminaire dans l'affaire Germain Katanga et Matthieu Ngudjolo a estimée qu'il existe deux situations de manque de volonté : la première est celle qui se manifeste par le souhait d'entraver le cours de la justice. La seconde est celle dans laquelle un État souhaite soustraire une personne pour plusieurs raisons, notamment, pour que la CPI n'exerce pas sa compétence à son égard. La seconde forme, non explicitement prévue par l'article $17 \mathrm{du}$ Statut, correspond au souhait de ne pas voir la personne traduite en justice ${ }^{59}$. Ce critère n'est pas un test de la bonne-foi des autorités nationales ${ }^{60}$ comme le pensent certains auteurs, mais plutôt celui de responsabilité d'un Etat à poursuivre les crimes pour lesquels, il a pleinement compétence en vertu de ses propres lois nationales. Toutefois, il incarne un élément subjectif quand il s'agit d'évaluer la qualité de la justice à la lumière des intentions réelles des États ${ }^{61}$. La subjectivité vise en réalité l'intérêt dans le chef de l'Etat de refuser d'agir en répression, puisqu'il s'agit ici d'une volonté politique que judiciaire.

Eu égard à cet intérêt, on peut distinguer deux cas d'espèces : D'une part, une procédure judiciaire fondamentalement creuse, qui laisse entrevoir son issue très favorable à l'accuse dont le

\footnotetext{
${ }^{57}$ Article 21-14è de loi n ${ }^{\circ}$ 15/024 du 31 décembre 2015 précitée : Les demandes d'arrestation aux fins de remise délivrées par la Cour sont adressées au Procureur général concerné, dans les formes prévues à l'article 21 ter ci-dessus

${ }_{58}$ Article 21-14 et Article 21-15e de loi no 15/024 du 31 décembre 2015 précitée.

59 Jacques MBOKANI, "L'impact de la stratégie de poursuite du Procureur de la CPI sur la lutte contre l'impunité et la prévention des crimes de droit international", à l'Université catholique de Louvain 2009, article disponible sur le site des Droits Fondamentaux: Cette interprétation a été confirmée par la Chambre d'appel de la même CPI.

${ }^{60}$ Almoktar ASHNAN, Op.Cit., 110.

61 Hans-Peter KAUL, "THE INTERNATIONAL CRIMINAL COURT: CURRENT CHALLENGES AND PERSPECTIVES”, Washington University Global Studies Law Review 6, no. 3 (2007): 575-582, 577.
} 
but dissimilé serait en outre, de faire prévaloir le principe : ne bis in idem en vue de décliner, in tempore opportuna, la compétence de la Cour. D'autre part, une décision étatique de nature politicoadministrative constituant une sorte d'armure contre toute procédure judiciaire. Il est évident que, la plupart de ces crimes ont toujours un lien plus ou moins politique, si leurs auteurs ne sont pas dans le fonctionnariat, ils seraient par contre ceux qui combattent le pouvoir en place afin d'obtenir un gain généralement quelconque. A ce titre, on peut évoquer le cas $\boldsymbol{B}$. Ntaganda, auquel, la CPI avait commencé à s'y intéresser un peu plus tôt jusqu'à délivrer en 2006 un mandat d'arrêt sous scellés à son encontre. Pourtant en 2009, dans le cadre d'un accord de paix, le gouvernement congolais l'avait nommé général au sein de l'armée congolaise. Il est demeuré à ce poste jusqu'à ce qu'il prenne fuite parmi d'autres militaires et crée avec eux en 2012 le groupe rebelle M23. S'il est vrai que le déclenchement des poursuites judiciaires au niveau national avaient été, par cette décision, impossibles, il saute aux yeux que la décision prise par l'Etat de le nommer au sein de l'armée congolaise à un poste militaire de haute responsabilité, n'avait que pour but, sous prétexte d'un accord de paix, de soustraire l'intéressé d'une quelconque justice notamment, internationale.

Ainsi, sous cet aspect, le manque de volonté couvre une assurance de l'impunité sous la forme d'une juridicité, tandis que la complémentarité de la Cour surgit, telle une garante contre cette impunité, que certains Etats ont toujours réservée à ces grands criminels. C'est dans ce sens, que malgré cette nomination, le procureur de la CPI n'avait cessé de prévaloir la compétence de la Cour et d'exiger l'arrestation et le transfèrement de l'intéressé.

Deuxièmement, la procédure doit avoir subi un retard injustifié qui, dans les circonstances, est incompatible avec l'intention de traduire en justice la personne concernée. Estimant difficile la prouvabilité de l'hypothèse précédente, les rédacteurs du Statut de Rome ont donc convenu d'ajouter ce second critère, afin de faciliter l'application de la complémentarité. En fonction des critiques, l'adjectif "injustifié" a été préféré à l'adjectif 'excessif', donnant ainsi à l'État la possibilité d'expliquer la raison pour laquelle, il a pris du retard. Ce critère rend l'évaluation plus objective ${ }^{62}$ que dans le premier cas. Ainsi, la Cour, examinant cette hypothèse, peut s'en tenir aux informations que l'Etat concerné pourrait avoir portées à son attention, conformément à la règle 51 du règlement de procédure et de preuve selon lesquelles, il démontre le défaut de pertinence dudit retard sur les principes juridiques internationalement admis.

Il est donc ici question d'une similarité dans le traitement des affaires au niveau interne, car il est possible d'établir le constat d'un retard injustifié, lorsque ceci est accompagné d'une intention d'échapper à la justice, avec les procédures habituelles de l'État dans des cas graves similaires ${ }^{63}$. Certes, de cette comparaison peut résulter une constatation de manœuvres dilatoires tendant à décourager voire, à suborner ou éliminer les témoins, corrompre les victimes ou toute personne susceptible de porter à la connaissance des autorités judiciaires un quelconque élément de preuve. Toutefois, même si la célérité d'un cas peut élucider sur la lenteur d'un autre, il est de bonne politique de ne pas considérer ce critère in globo, pour autant que chaque affaire comporte ses particularités. Il conviendrait par conséquent, d'enquêter sur les circonstances de chaque situation séparément, afin de déterminer si, selon les faits d'un cas donné, l'intention était d'éviter que l'accusé soit face à la justice. $^{64}$

Enfin troisièmement, il doit être prouvé, que la procédure n'a pas été ou n'est pas menée de manière indépendante ou impartiale mais d'une manière qui, dans les circonstances, est incompatible avec l'intention de traduire en justice la personne concernée. S'agissant de l'impartialité et l'indépendance, le statut ne précise pas ce que l'on entend, en son sens, par ces notions, la doctrine renseigne que, l'impartialité est un état d'esprit de celui guidé par le souci de la justice en se référant au droit. Elle implique une absence d'opinion préconçue, de parti pris, de préjugés ${ }^{65}$. Bref, elle réfute tout élément ne se rapportant pas à la cause, lors de l'appréciation des

\footnotetext{
${ }^{62}$ Almoktar ASHNAN, Op.Cit., 110.

${ }^{63}$ Ibid.

${ }^{64}$ Mohamed El ZEIDY, The principle of complementarity in international criminal law: origin, development and practice (Leiden: 1ère éd., Boston, 2008) 182. Cité par A. ASHNAN, Loc.Cit.

${ }^{65}$ Télesphore KAVUNDJA N. MANENO, "L'INDEPENDANCE ET L'IMPARTIALITE DU JUGE EN DROIT COMPARE BELGE, FRANÇAIS ET DE L'AFRIQUE FRANCOPHONE”, L'impartialité, thèse de doctorat en droit, U.C.L., Louvain-la-Neuve 2, (2005): 262-263.
} 
faits. Tandis que, l'indépendance ${ }^{66}$ qui entretient des rapports très étroits avec la première, consiste en une absence de toute influence d'autres pouvoirs notamment, législatif et exécutif sur le cours procédural.

Dans le premier cas, la notion inclut bien à la fois le procureur et le juge, alors que dans le second, l'attention sera plus focalisée sur le travail du parquet, dans son pouvoir de déclencher les poursuites. Puisqu'en effet, le lien de subordination qui existe entre le parquet et le pouvoir exécutif à travers $l e$ ministre de la justice ou de la défense, ne peut garantir au procureur chargé des poursuites, une pleine indépendance. Or, en cette matière, l'importance de son indépendance est indispensable dans la mesure où, il représente le seul organe habilité d'intenter l'action en justice, au cas où, il s'agirait de personnalités politiques bénéficiant du privilège de juridiction. En droit congolais par exemple, il n'existe aucune possibilité pour le juge de se saisir d'office en cette matière, et la victime ne peut pas le saisir par citation directe ${ }^{67}$. Ce qui ouvre largement la voie à l'influence politique, qui est toujours aux aguets. Il convient de scruter objectivement l'attitude dudit procureur à l'égard des positions et avis purement politiques des membres du gouvernement, afin de mieux apprécier son indépendance.

Dans tous les cas, l'appréciation de la volonté de l'Etat d'enquêter et de poursuivre penche visiblement vers une justice plus rigoureuse à l'encontre du suspect, excluant toute faveur illégale et inique à son bénéfice, tendant à lui éviter le poids d'un procès. Cependant, il n'est nullement question de priver au suspect de son droit à un procès équitable peu importe la gravité des faits invoqués.

\section{b. Appréciation de l'incapacité de l'Etat à poursuivre}

En appréciant l'incapacité d'un Etat à enquêter et à poursuivre, la complémentarité se constitue en un élément qui éprouve le système judiciaire national. Mais il faut bien en prendre garde parce que, la Cour ne peut pas porter un jugement sur les actes juridictionnels de la justice nationale dans les procédures qu'elle a engagées. La Cour constate plutôt une situation selon laquelle, le fonctionnement de cette justice n'est pas régulier et rassurant face aux enjeux que comporte généralement un procès pour crimes internationaux. Ainsi, l'incapacité de l'Etat se rapporte d'un côté à l'effondrement de la totalité ou d'une partie substantielle de son appareil judiciaire, et de l'autre côté, à l'indisponibilité de celui-ci, de se saisir de l'accusé, de réunir les éléments de preuve et les témoignages nécessaires ou de mener autrement à bien la procédure. ${ }^{68}$

Concernant l'effondrement, le statut ne précisant pas le sens qu'il entend donner à cette expression, certains auteurs estiment que l'effondrement est total, lorsqu'il y a destruction complète de l'administration de la justice, soit en raison de la perte par l'État de son contrôle sur le territoire ou en raison d'une décision nationale de supprimer l'administration de la justice. ${ }^{69} \mathrm{Et}$, un effondrement substantiel comprend une situation, où les autorités de l'État ne sont pas nécessairement dysfonctionnelles, mais tout de même incapable d'assurer l'instruction de l'affaire ${ }^{70}$. L'adjectif substantiel, avait été préféré à l'adjectif "partiel", initialement utilisé par la commission préparatoire, en vue de renforcer la priorité de la juridiction nationale et d'éviter que la Cour assume la compétence du seul fait d'un conflit armé existant dans un État ${ }^{71}$. En pratique, les éléments tels que, le manque de personnel nécessaire et ou d'infrastructure judiciaire, l'absence d'une législation pénale, l'amnistie des criminels par le pouvoir politique ${ }^{72}$, peuvent être analysés comme éléments d'appréciation de l'effondrement d'un système judiciaire.

\footnotetext{
${ }^{66}$ C'est la situation du juge auquel son statut (dispositions constitutionnelles et légales) assure la possibilité de prendre ses décisions à l'abri de toutes les influences, instructions et pressions. Georges DE LEVAL, Institutions judiciaires (Liège: Collection Scientifique de la Faculté de Droit de Liège, 1993) 37.

${ }^{67}$ Article 54 al. 2 du DÉCRET du 6 août 1959 portant le Code de procédure pénale telle que modifié par la loi no 15/024 du 31 décembre 2015 précitée.

${ }^{68}$ Communication relative à certaines questions de politique générale concernant le Bureau du Procureur. Document en ligne sur: https://www.icc-cpi.int/NR/rdonlyres/1FA7C4C6-DE5F-42B7-8B25 60AA962ED8B6/143595/030908_Policy_Paper_FR.pdf. (Consulté le 30/01/2019).

69 Jo STIGEN, The relationship between the International Criminal Court and National Jurisdictions (the principle of complementarity) (Boston: Ledien, 2008) 314. Cité par A. ASHNAN, Loc.Cit..

${ }^{70}$ Jurdi NIDAL NABIL, "THE PROSECUTORIAL INTERPRETATION OF THE COMPLEMENTARITY PRINCIPLE: DOES IT REALLY CONTRIBUTE TO ENDING IMPUNITY ON THE NATIONAL LEVEL?”, International Criminal Law Review 10, (2010): 73-96, 80, DOI: 10.1163/157181209x12584562670857.

${ }^{71}$ Holmes (J.), Complementarity: National Courts versus the ICC, The Rome Statute of the International Criminal Court, A commentary, Op.Cit., 677.

72 JURDI Nabil (N.), Op.Cit., 230.
} 
S'agissant de l'indisponibilité, à défaut d'une définition statutaire, la doctrine laisse comprendre qu'il existe bien dans ce cas un système judiciaire qui fonctionne, mais qui a échoué ${ }^{73}$, dans la mise en œuvre effective des procédures qui concourent à une bonne organisation du procès. Il est ici question du succès de l'investigation et de poursuites, qui dépend de différents moyens appropriés notamment, matériels, financiers, juridiques etc., susceptibles de produire les meilleurs résultats ou d'assurer l'efficacité de l'action judiciaire.

L'ancienne Procureur du TPIY, Louise ARBOUR, avait critiqué le régime de complémentarité en arguant qu'il favoriserait les pays riches et développés au détriment des États pauvres ${ }^{69}$. Un argument pratique et réaliste mais qui s'écarte quelque peu de l'explication fondamentale de ce principe, dans la mesure où, tous les Etats sont ou devraient être égaux et aucun ne peut travailler à la place de l'autre. D'ailleurs, ces Etats que l'on estime pauvres, ne tergiversent pas à brandir la souveraineté pour réfuter une action de la Cour. A cet effet, faut-il rappeler que la souveraineté c'est la bonne gouvernance et celle-ci résulte de la responsabilisation. Ainsi, la Cour qui ne supplante pas les Cours et Tribunaux des Etats, les invite, à travers la complémentarité, à capabiliser leurs systèmes judiciaires dans la recherche de ces crimes, l'arrestation des suspects, la collecte d'éléments de preuve, la prise en charge des victimes etc. Bref, l'Etat doit assumer sa responsabilité, qui est d'ailleurs, liée à son existence entant que tel, parce qu'un Etat sans justice est inexistant.

\section{c. Conséquence de la décision de la Cour d'intervenir dans un Etat: La complémentarité comme élément déclencheur des procédures nationales}

Les rédacteurs de cet article lui ont assigné quatre objectifs: se focaliser une fois de plus sur l'importance capitale de la complémentarité de la CPI; encourager et permettre aux Etats d'exercer très tôt leur droit d'enquête et de poursuite; éviter ainsi des enquêtes ou des procédures parallèles; et enfin en faire un outil de contrôle ou de limite des pouvoirs du Procureur de la $\mathrm{CPI}^{70}$. Cette disposition dans son esprit, parait éminemment explicative, démontrant la praticabilité de la complémentarité et qui donne l'image d'un gendarme-juridictionnel derrière les Etats, leur rappelant à chaque fois leurs obligations d'agir en répression et en prévention contre ces crimes internationaux. Sa teneur permet à la Cour de combattre dans la mesure du possible, toute volonté politique favorable à l'impunité, tel qu'il a été illustré dans l'affaire B. Ntaganda susmentionnée.

Cette obligation de notification prend la forme d'une véritable mise en demeure faite audit Etat en vue d'assumer sa responsabilité. C'est une forme de déclenchement des poursuites nationales en avertissant d'une intervention internationale en vue. Ainsi, cette signification aura pour conséquence directe, d'une part, la réaction de l'Etat qui pourrait dans le mois qui suit sa réception, informer la Cour qu'il ouvre ou a ouvert une enquête sur ses ressortissants ou d'autres personnes sous sa juridiction pour des actes criminels qui pourraient être constitutifs des crimes visés à l'article $5 \mathrm{du}$ statut, et qui ont un rapport avec les renseignements notifiés. S'il le lui demande, le Procureur lui défère le soin de l'enquête sur ces personnes. D'autre part, elle permet dans le cas où le Procureur concède (sans recourir à la chambre préliminaire), d'établir une coopération étrécie entre la Cour et l'Etat concerné dans le cadre de ladite affaire.

\section{Conclusion}

Après plus de deux décennies, force est de relever l'importance de la CPI dans le système pénal international dans la lutte pour la paix et la sécurité de l'humanité. Sa complémentarité qui justifie sa permanence vise la lutte contre toute impunité que les systèmes nationaux pourraient réserver aux criminels qui leur présentent un certain intérêt. D'où, l'origine de cette hostilité presque irréconciliable envers cette Cour qui d'ailleurs, était prévisible depuis la période des discussions relatives à sa création. Cependant, du nombre d'affaires pour lesquelles la Cour a utilisée sa compétence, il ressort que la complémentarité n'est pas en soi un problème. Parce que d'une part, les Etats ont majoritairement recouru à la compétence de la Cour et d'autre part, le conseil de sécurité qui comprend les Etats puissants et hostiles à cette juridiction, n'a pas hésité à y recourir pour tenter de suppéditer la paix (théoriquement) dans l'une des régions du monde, notamment, au Darfour et

\footnotetext{
${ }^{73}$ LAUCCI (C.), "Les compétences nationales et internationales en matière de répression des crimes de guerre”, thèse de doctorat, Aix-Marseille III, (2001), 975.

${ }^{69}$ LAUCCI (C.), Op.Cit., 977.

${ }^{70}$ A. ASHNAN, Op.Cit., 201.
} 
en Lybie. Ceci implique que les Etats et la communauté internationale reconnaissent à la Cour une certaine capacité de suppléer un vide judiciaire dans une partie du monde.

Voyons par exemple la Libye qui n'est pas partie au traité et dont la situation a été transférée par le conseil de sécurité, a fourni un très bel exemple, celui de ne pas décrier la Cour, mais d'invoquer devant ses juges, qu'en vertu de la complémentarité de la Cour, celle-ci ne devait pas agir en lieu et place de l'Etat libyen, car son système était bien capable et il y avait la volonté de poursuivre. Il en est de même de ceux des Etats qui collaborent sans difficulté avec la Cour, dont le cas kenyan ayant impliqué le Président Uhuru Kenyatta et son Vice-président William Ruto. ${ }^{71}$ Cette attitude qui semblerait être une impuissance de l'Etat, comme on le dit toujours dans le langage commun : "que la CPI est une juridiction contre les Etats faibles", révèle en réalité la volonté (consciente ou inconsciente) de bâtir un monde relativement paisible et sécurisé selon les normes que les Etats eux-mêmes décident souverainement de mettre en place au niveau international. On ne peut prétendre vouloir la paix et ignorer la justice, et celle-ci symbolisant la cohésion sociale, exigent préalablement un degré d'adhésion collective, afin de répandre son influence dissuasive sur un large spectre.

Cependant, reconnaitre la compétence de la Cour, c'est avant tout reconnaitre l'obligation de rendre son système interne capable et de briser toute barrière de quelque nature que ce soit, susceptible d'étouffer une action judiciaire ou de torpiller sa substance. Car, on peut le rappeler, la Cour mesure son efficacité par l'absence d'affaires à son niveau, parce que les Etats eux-mêmes agissent congrument.

A. Journal

Bibliography

BAMEME, Bienvenu WANE. "LA QUESTION DE JURIDICTIONS CONGOLAISES COMPETENTES EN MATIERE DE CRIMES CONTRE LA PAIX ET LA SECURITE DE L'HUMANITE", Revue de l'université congolaise I, 57, 2017: 55-83.

BECKER, Steven W. "LES OBJECTIONS DES GRANDES NATIONS A LA COUR PENAL INTERNATIONALE", ERES “Revue internationale de droit pénal” 81, no. 1, 2010: 47-64.

CURRAT, Philippe. "HANS KELSEN, CARL SCHMITT ET LA COUR PENALE INTERNATIONALE. QUELQUES REFLEXIONS APRES LA PREMIERE DECENNIE D'EXERCICE DE LA COUR', AFRI 14, 2013: 497-525.

DECHERF, Lire Dominique. "LE KENYA ET LA COUR PENALE INTERNATIONALE", Études 11 Tome 419, 2013: 449-460.

DEZALAY, Sara. "L'AFRIQUE CONTRE LA COUR PENALE INTERNATIONALE ? ÉLEMENTS DE SOCIOGENESE SUR LES POSSIBLES DE LA JUSTICE INTERNATIONALE", Editions Karthala Politique africaine 156, no. 2, 2017: 165-182.

Jurdi, NIDAL NABIL, "THE PROSECUTORIAL INTERPRETATION OF THE COMPLEMENTARITY PRINCIPLE: DOES IT REALLY CONTRIBUTE TO ENDING IMPUNITY ON THE NATIONAL LEVEL?", International Criminal Law Review 10, 2010 : 73-96, DOI: 10.1163/157181209x12584562670857.

KAUL, Hans-Peter. "THE INTERNATIONAL CRIMINAL COURT: CURRENT CHALLENGES AND PERSPECTIVES”, Washington University Global Studies Law Review 6, no. 3, 2007: 575-582.

KHAMIDOVA, M. F. "CREATION D'UNE COUR PENALE INTERNATIONALE ET QUESTIONS JURIDIQUES DE RESPONSABILITE POUR LES CRIMES INTERNATIONAUX", Bulletin de l'Université d'État de Saint-Pétersbourg 3, no. 8, 2017: 337348.

ORENTLICHER, Lire aussi., Diane F, "POLITIQUE PAR D'AUTRES MOYENS: LE DROIT DE LA COUR PENALE INTERNATIONALE”, Cornell International Law Journal 32, no. 3, 1999.

\footnotetext{
${ }^{71}$ Lire Dominique DECHERF, “LE KENYA ET LA COUR PENALE INTERNATIONALE”, Études 11 Tome 419 (2013): 449-460.
} 
TOUSIGNANT, Maxime C.- "L'INSTRUMENTALISATION DU PRINCIPE DE COMPLEMENTARITE DE LA CPI: UNE QUESTION D'ACTUALITE”, Revue Québécoise de droit international 25, no. 2, 2012: 73-99.

\section{B. Livre et Thèse}

Almoktar ASHNAN, "Le principe de complémentarité entre la cour pénale internationale et la juridiction pénale nationale", Thèse, Faculté de Droit/Droit public, Université François Rabelais de Tours, 2015.

DE LEVAL, Georges. Institutions judiciaires. Liège: Collection Scientifique de la Faculté de Droit de Liège, 1993.

El ZEIDY, Mohamed. The principle of complementarity in international criminal law: origin, development and practice. Leiden: 1ère éd., Boston, 2008.

GRANGÉ, Ninon (dir.), RAMEL, Frédéric (dir.), Le droit international selon Hans Kelsen : Criminalités, responsabilités, normativités. Nouvelle édition [en ligne], Lyon: ENS Éditions, 2018.

Jacques MBOKANI, "L'impact de la stratégie de poursuite du Procureur de la CPI sur la lutte contre l'impunité et la prévention des crimes de droit international", à l'Université catholique de Louvain 2009.

LAUCCI (C.), "Les compétences nationales et internationales en matière de répression des crimes de guerre", thèse de doctorat, Aix-Marseille III, 2003.

MANENO, Télesphore KAVUNDJA N. "L'indépendance et l'impartialité du juge en droit comparé belge, français et de l'Afrique francophone", L'impartialité, de doctorat en droit, U.C.L., Louvain-la-Neuve 2, 2005: 262-263.

R KAYUMOVA, Alfiya. La juridiction pénale en droit international. Kazan: Centre d'innovation technologique, 2016.

SEILS, Paul. Guide de la complémentarité: Introduction quant au rôle des juridictions nationales et de la Cour pénale internationale dans la poursuite des crimes internationaux. ICTJ: Meredith Barges, 2016.

STIGEN, Jo. The relationship between the International Criminal Court and National Jurisdictions (the principle of complementarity). Boston: Ledien, 2008.

TRIFFTERER, Otto. Commentaire du Statut de Rome de la Cour pénale internationale, Observations, notes article par article, 1 ère éd. Bruxelles: Bruylant, 1999.

\section{Règlements}

Affaire: Le Procureur $c$. Jean-Pierre Bemba Gombo.

Conférence de l'Union Africaine, 13e session ordinaire, Syrte, 3 juillet 2009, Décision sur le rapport de la réunion des États africains parties au Statut de Rome de la Cour pénale internationale (C.P.I.) - Assemblée/AU/Déc., 245 (XIII) Rev.1, § 10.

J.O.RDC., $52^{2 \mathrm{ème}}$ année, $\mathrm{n}^{\circ}$ spécial du 05 février 2011.

La loi de corroborée par de la loi no 15/023 du 31 décembre 2015 modifiant la loi no 024-2002 du 18 novembre 2002 portant Code pénal qui, dans le deuxième paragraphe de son préambule soutient que, la reconnaissance par la loi organique de 2013, aux juridictions de droit commun, la compétence de connaitre de ces crimes, leur eut perdu le caractère d'infraction exclusivement militaire.

La loi n⿳024/2002 du 18 novembre 2002 portant Code pénal militaire

la loi nº9/001 du 10 janvier 2009 portant protection de l'enfant, in JO RDC, 50è année, n spécial, Première partie, du 12 janvier 2009

La République démocratique du Congo a ratifié cette Convention internationale à travers son Décret $\mathrm{n}^{\circ} 0013 \mathrm{du} 30 / 03 / 2002$.

Loi ${ }^{\circ} 023 / 2002$ du 18 novembre 2002 portant Code judiciaire militaire

Loi $n^{\circ} 15 / 024$ du 31 décembre 2015.

Loi organique $\mathrm{n}^{\circ} 13 / 011-\mathrm{B}$ du 11 avril 2013 portant organisation, fonctionnement et compétences des juridictions de l'ordre judiciaire, in JO RDC,54ième année, $\mathrm{n}^{\circ}$ spécial-Première partie, du 04 mai 2013. 
Rapport du Haut-Commissariat des Nations Unies aux droits de l'homme du Conseil des droits de l'homme Groupe de travail sur l'Examen périodique universel, Vingt-septième session 1 er -12 mai 2017, Compilation concernant les Philippines, 3.

RéférenceC.N.138.2018.TREATIESXVIII.10//https://treaties.un.org/doc/publication/cn/2018/cn.13 8.2018-eng.pdf

Report of the ad hoc Committee on the Establishement of an International Criminal Court// Document de l'ONU du 06/09/1995.

Statut de Rome.

Voir l'article dans le magazine LE TEMPS, Publié lundi 10 septembre 2018 à 19:51, modifié lundi 10 septembre 2018 à 19:54.

Voir les travaux de la Conférence de révision du Statut de Rome, tenue à Kampala, Ouganda, juin 2010, Complémentarité, RC/11, annexe V(c), Résumé officieux des points focaux, et autres documents liés, disponible sur le site Internet www.icc-cpi.int/iccdocs/asp_docs/RC2010/RC11-Annex.V.c-FRA.pdf. Cf. également les Rapports du Bureau et du Secrétariat de l'Assemblée des Etats parties sur la complémentarité, présentés à la $11^{\text {ème }}$ session de l'Assemblée, La Haye, Pays-Bas, 14-22 nov. 2011, respectivement ICC-ASP/11/24 et 25.

\section{Internet}

http://www.elibrary.az/docs/jurnal/jrn2017_731.pdf, consulté le 20/01/2019.

https://treaties.un.org/doc/Publication/CN/2016/CN.805.2016-Eng.pdf, Consulté le 30/01/2019. https://treaties.un.org/doc/Publication/CN/2016/CN.862.2016-Eng.pdf, Consulté le 30/01/2019. https://www.icc-cpi.int/about?ln=fr, Consulté le 30/01/2019.

https://www.icc-cpi.int/NR/rdonlyres/1FA7C4C6-DE5F-42B7-

8B2560AA962ED8B6/143595/030908_Policy_Paper_FR.pdf, Consulté le 30/01/2019. https://www.icc-cpi.int/NR/rdonlyres/1FA7C4C6-DE5F-42B7-

8B2560AA962ED8B6/143595/030908_Policy_Paper_FR.pdf, Consulté le 30/01/2019. https://www.icc-cpi.int/NR/rdonlyres/1FA7C4C6-DE5F-42B7-

8B2560AA962ED8B6/143595/030908_Policy_Paper_FR.pdf, Consulté le 30/01/2019. https://www.icc-cpi.int/Pages/item.aspx?name=180208-otp-stat\&ln=fr ，Consulté le 30/01/2019. https://www.icc-cpi.int/Pages/item.aspx?name=pr1034\&ln=fr, Consulté le 30/01/2019. 
